\title{
Research on the Anti-interference of Intelligent Vehicle Path Tracking on Variable Working Conditions Based on $\mathrm{H} \infty$ Control
}

\author{
Chuanyin Tang ${ }^{1,{ }^{*}}$, Yifeng Zhao ${ }^{2}$, Yafeng Zhao ${ }^{3}$ and Shuwen Zhou ${ }^{4}$ \\ ${ }^{1}$ School of Mechanical Engineering \&Automation, Northeastern University, Shenyang 110819, \\ China \\ ${ }^{2}$ Shanghai Superior Die Technology CO.,Ltd., Shanghai \\ ${ }^{*}$ Corresponding author
}

\begin{abstract}
Keywords: Intelligent vehicles, Anti-interference, Path tracking control, Vehicle model, Lateral displacement deviation.
\end{abstract}

\begin{abstract}
Based on $\mathrm{H} \infty$ control theory, the path tracking control of intelligent vehicle is proposed in the paper. Considering the possible interference of various factors in the path tracking process, the controller is designed to make the whole system have good anti-interference ability and robustness. The control effect of intelligent vehicle at different speeds and under different road adhesion coefficients is presented. The results show that, under the disturbance of lateral wind and road changes, with different speeds and different road adhesion coefficients, the lateral displacement deviation decreases gradually and the final deviation converges to zero. The change of yaw angle and yaw velocity tends to be stable finally. This indicates that, under the action of the controller proposed, the intelligent vehicles can well resist external interference in the process of path tracking, on variable working conditions with different speeds and different road adhesion coefficients, and have good stability in the process of driving.
\end{abstract}

\section{Introduction}

The concept of intelligent cars has been gradually known by more and more people in recent years, and the related technologies have been widely used. People have a new understanding of the traditional car, the form of driving the car has slowly changed. Intelligent vehicles can automatically complete driving behaviors in daily traffic through advanced technologies such as environmental awareness technology, electronic information technology and intelligent control. Intelligent vehicle study field is very wide, it is not only a set of environmental perception, decision-making and path planning and motion control technology in the integration of intelligent driving system, it is a by automatic control, electronic communications, computer, artificial intelligence and deep learning complex composed of multiple disciplines and cutting-edge technology. The system architecture involving key technologies of intelligent vehicles is shown in Figure 1. Motion control technology generally includes longitudinal control and transverse control, in which the transverse control is mainly to control the vehicle's path tracking ability, and the longitudinal control is mainly to control the vehicle's running speed or acceleration.

Lateral control is an important part of intelligent vehicle motion control technology and a good lateral control algorithm is the basic guarantee for intelligent vehicle to complete path tracking. Scholars have made a lot of contributions to the lateral motion control of intelligent vehicles, as in [1-5]. Reference [4] aiming at the problem of performance degradation caused by system uncertainty in the process of lateral maneuver of unmanned vehicles, a sideshow stability control method based on $\mu$ integrated method was proposed under cascade control structure. Intelligent vehicle in the process of path to follow, under lateral wind disturbance, and road curvature change under the action of the controller, in view of the different speed and different road adhesion coefficient change condition, intelligent vehicle to follow the path that could be affected by various factors in the process of interference, such as vehicles running parameters change, the process of change of stroke, different road conditions, etc. Therefore, in the study of vehicle path following control, the 
interference of various factors such as air resistance and road curvature change during the path following process should be considered to make the whole system have good robustness.

The influence and control effect of intelligent vehicles on path following when they are disturbed by lateral wind and road curvature changes under varying working conditions of different speeds and road adhesion coefficients are discussed in the paper.

\section{Model of Vehicle Dynamics}

The vehicle dynamics model was established and the following idealized assumptions were made: assume that the vehicle did not sharply turn the steering wheel significantly; Assuming that the suspension system and the vehicle are rigid, only the lateral motion and yaw motion of the vehicle are considered, ignoring the influence of the vehicle's pitch motion and roll motion; Suppose the vehicle is driving on a flat road; Assume the car's forward speed is constant; It is assumed that the lateral characteristic of tire is in the linear range, and the left and right transfer of load is not considered. Assume that the vehicle is unaffected by both longitudinal and transverse aerodynamics.

Within time $\Delta t$, get the deviation of the front wheel of the vehicle to the reference track from the kinematic relation of the vehicle:

$$
\dot{y}_{f}=\sqrt{u^{2}+v^{2}} \sin (\beta+\varphi)+\operatorname{lr}=V(\beta+\varphi)+l r
$$

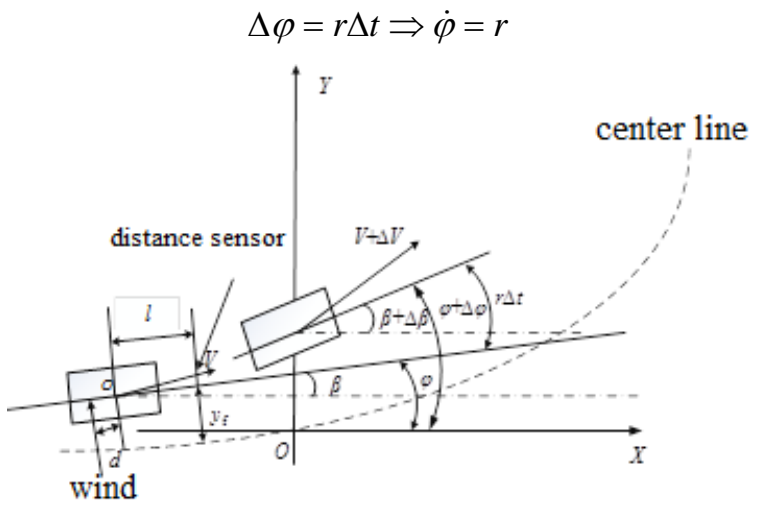

Figure 1. Model of front wheel steering vehicle

Vehicle dynamics equation:

$$
\begin{gathered}
{\left[\begin{array}{c}
\dot{\beta} \\
\dot{r} \\
\dot{\varphi} \\
\dot{y}_{f}
\end{array}\right]=\left[\begin{array}{cccc}
a_{11} & a_{12} & 0 & 0 \\
a_{21} & a_{22} & 0 & 0 \\
0 & 1 & 0 & 0 \\
V & l & V & 0
\end{array}\right]\left[\begin{array}{c}
\beta \\
r \\
\varphi \\
y_{f}
\end{array}\right]+\left[\begin{array}{c}
b_{11} \\
b_{21} \\
0 \\
0
\end{array}\right] \delta+\left[\begin{array}{cc}
0 & d_{1} \\
0 & d_{2} \\
0 & 0 \\
0 & 0
\end{array}\right]\left[\begin{array}{ll}
\rho & F_{w}
\end{array}\right] \quad a_{11}=\frac{k_{f}+k_{r}}{m u}, a_{12}=-1+\frac{a k_{f}-b k_{r}}{m u^{2}},} \\
a_{21}=\frac{a k_{f}-b k_{r}}{I_{Z} u}, a_{22}=\frac{a^{2} k_{f}+b^{2} k_{r}}{I_{Z} u} \\
b_{1}=-\frac{k_{f}}{m}, b_{2}=-\frac{a \cdot k_{f}}{I_{z}}, d_{1}=\frac{1}{m V}, d_{2}=\frac{d}{I_{Z}}
\end{gathered}
$$

The equation of state of the vehicle dynamics model is represented as:

$$
\left\{\begin{array}{c}
\dot{x}=A x+B_{1} u+B_{2} w \\
y=C x
\end{array}\right.
$$




$$
A=\left[\begin{array}{cccc}
a_{11} & a_{12} & 0 & 0 \\
a_{21} & a_{22} & 0 & 0 \\
0 & 1 & 0 & 0 \\
V & l & V & 0
\end{array}\right], B_{1}=\left[\begin{array}{c}
b_{11} \\
b_{12} \\
0 \\
0
\end{array}\right], B_{2}=\left[\begin{array}{cc}
0 & d_{1} \\
0 & d_{2} \\
0 & 0 \\
0 & 0
\end{array}\right], C=\left[\begin{array}{llll}
0 & 0 & 0 & 1
\end{array}\right]^{T}
$$

\section{Hळ Robust Controller}

Hळ control theory belongs to modern control theory and can solve the robust stability problem of the system described by $\mathrm{H} \infty$. The linear matrix inequality theory is applied to analyze the $\mathrm{H} \infty$ control.

The vehicle system state equation is described as:

$$
\dot{x}(t)=A x(t)+B_{1} u(t)+B_{2} w(t)
$$

Where, the system state variable is:

$$
x(t)=\left[\begin{array}{llll}
\beta & r & \varphi & y_{f}
\end{array}\right]^{T}
$$

The disturbance quantity of the system is:

$$
w(t)=\left[\begin{array}{ll}
\rho & F_{w}
\end{array}\right]^{T}
$$

System control input is:

$$
u(t)=\delta
$$

The system matrix, input matrix and system parameter matrix are matrix A, B1, B2 respectively. Under the action of $\mathrm{u}=\mathrm{Kx}$ the system satisfies $\mathrm{H} \infty$ stable performance index ${ }_{\tau}$ Among them:

$$
K=(P B)^{-1} X
$$

\section{Simulation Analysis}

The passenger car is selected as the specific research object. The previous wheel Angle is the control input, the disturbance is the changing road and lateral wind, and the output is the deviation of the front wheel relative to the reference path. In order to verify the intelligent vehicle under the action of the controller has a good anti-interference ability, and as far as possible in line with the actual driving process of the vehicle encountered. The control effects of intelligent vehicles under different speeds and road adhesion coefficients are analyzed.

The design of the controller was simulated and verified. The simulation software was Matlab\&Simulink, and the design of the simulation model, including reference trajectory model, controller and controlled object, was completed through MATLAB LMI Control Toolbox and sfunction. The interference value is rho ref of road curvature and lateral wind fw. According to the classification of wind speed grades by China meteorological administration, branches will swing and flags will flutter when the wind speed is between 8.0 and $10.0 \mathrm{~m} / \mathrm{s}$, which is a relatively common wind speed and belongs to category 5 wind. The range of lateral wind is presented as:

$$
F_{w}=\frac{1}{2} C_{D} A \rho_{0} u_{r}
$$

Where, is the air resistance coefficient; is windward area of vehicle; is air density; is the relative velocity. Road curvature of interference value pref and lateral wind fw is shown in Figure 2 and Figure 3. 


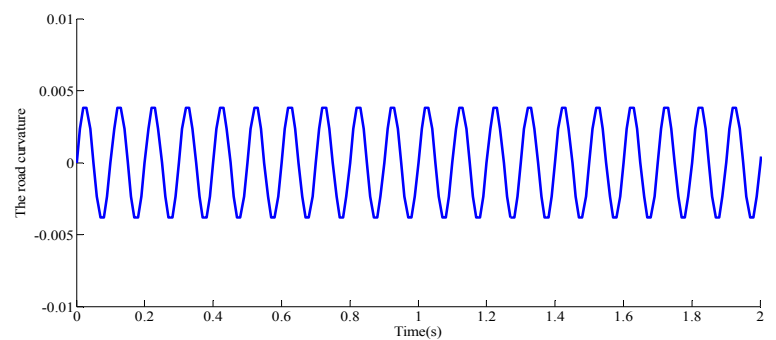

Figure 1. Curvature of the road

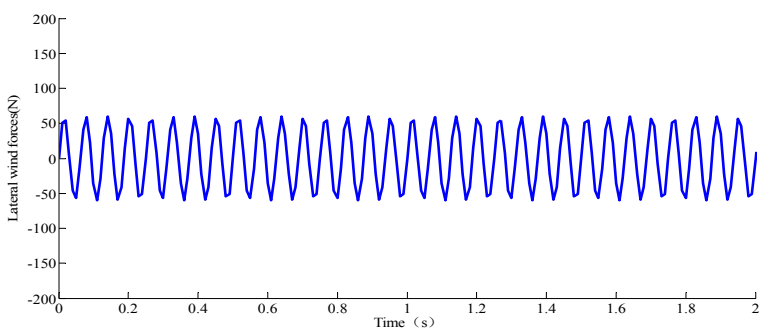

Figure 2. Lateral wind force

\section{Control Effect Analysis with Different Speeds}

The path tracking of vehicles at speeds of $10 \mathrm{~m} / \mathrm{s}$ and $20 \mathrm{~m} / \mathrm{s}$ is analyzed. Ability to resist interference along with the process. Figure 4 shows the change curve of lateral deviation of the vehicle, Figure 5 shows the change curve of front wheel Angle, and Figure 6 shows the change curve of lateral deviation of the center of mass.

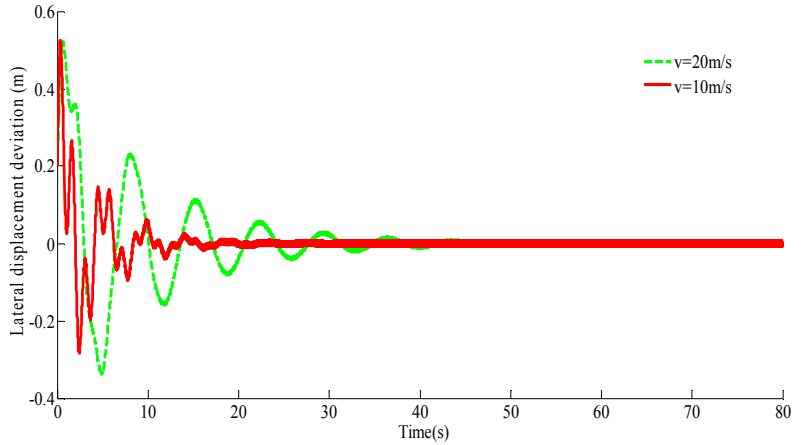

Figure 3. Comparison of lateral displacement deviation with different speeds

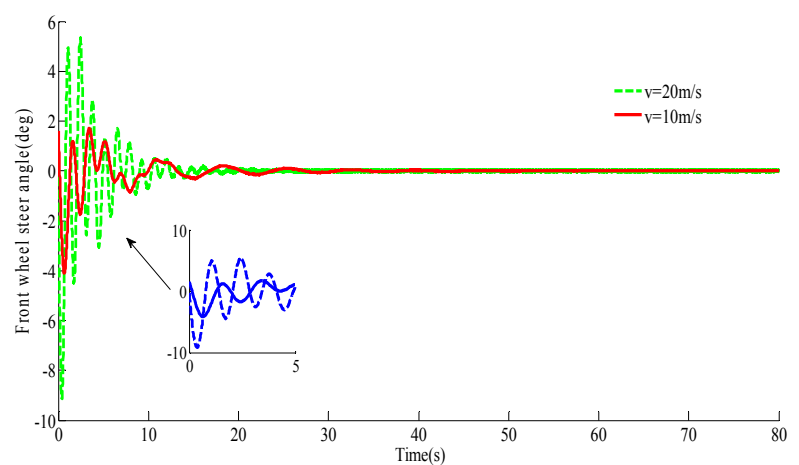

Figure 4. Comparison of front wheel steer angle with different speeds 


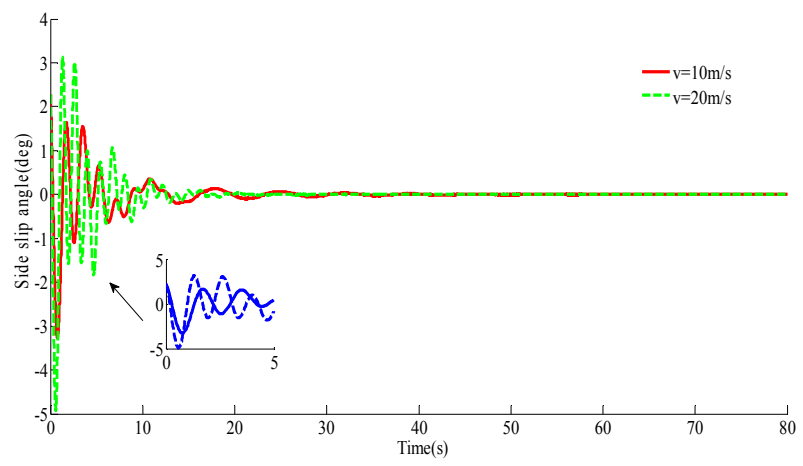

Figure 5. Comparison of side slip angle of center of mass with different speeds

According to Figure 4, with the increase of vehicle speed, the range of lateral deviation changes increases, but the lateral deviation can be corrected timely under the action of the controller. When the vehicle speed is $10 \mathrm{~m} / \mathrm{s}$, the change range is $-4 \mathrm{deg} \sim 1.8 \mathrm{deg}$; when the vehicle speed is $20 \mathrm{~m} / \mathrm{s}$, the change range is $-9 \mathrm{deg} \sim 5 \mathrm{deg}$. With the increase of the vehicle speed, the change of the front wheel Angle is relatively gentle. The variation amplitude of center-of-mass deviation Angle increases with the increase of velocity and eventually tends to be stable. It indicates that under the action of the controller, the vehicle has good anti-interference performance in the process of different speeds.

\section{Analysis of Control Effect with Different Controller under Different Adhesion Coefficients}

The simulation results are compared and analyzed under different adhesion coefficients. The road adhesion coefficients were 0.7 and 0.3 respectively, and the vehicle speed was $15 \mathrm{~m} / \mathrm{s}$. Figure 7 shows the change curve of lateral deviation of the vehicle, Figure 8 shows the change curve of front wheel Angle, and Figure 9 shows the yaw angular velocity.

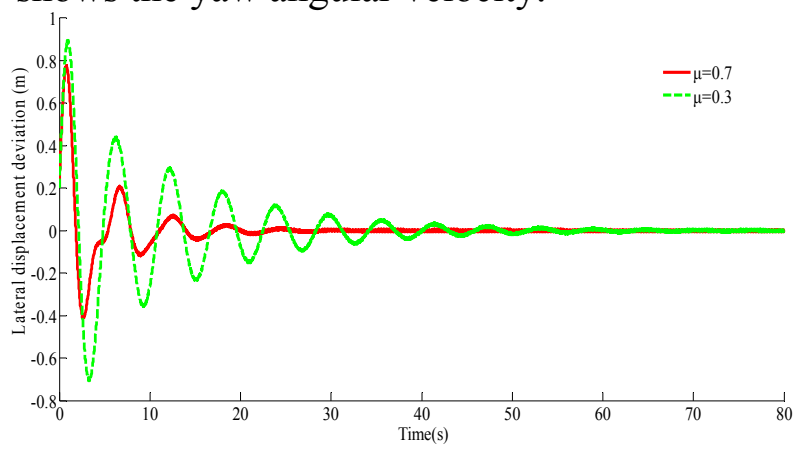

Figure 6. Comparison of deviation of lateral displacement with different adhesion coefficient

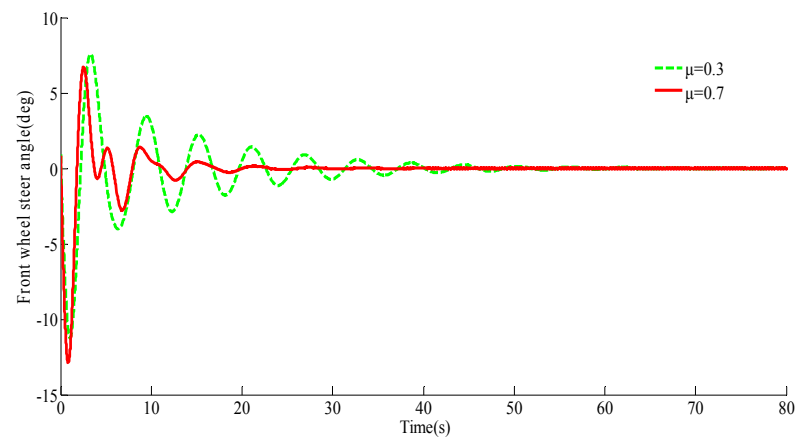

Figure 7. Comparison of front wheel steer angle with different adhesion coefficient 


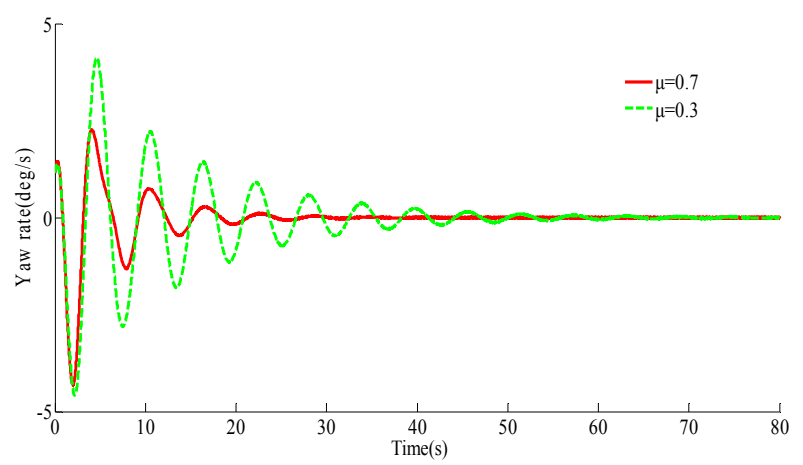

Figure 8. Comparison of angular velocity of yaw with different adhesion coefficient

According to Figure 7, the road adhesion conditions become worse, and the range of lateral deviation increases with the decrease of road adhesion coefficient. However, under the action of the controller, the lateral deviation is timely corrected, and finally converges to zero. The variation of the lateral deviation Angle of the center of mass and the yaw angular velocity is roughly consistent, and their variation amplitude increases with the decrease of the road adhesion coefficient, and finally tends to be stable. It indicates that under the action of the controller, the vehicle can resist the external interference in the process of following the path, and has a good stability in the process of driving.

\section{Conclusions}

The lateral deviation of the vehicle relative to the reference path decreases gradually and the final deviation converges to zero under the action of $\mathrm{H} \infty$ controller at different speeds; The lateral deviation can be corrected in time with the increase of velocity. It shows that intelligent vehicle can resist external interference well in the process of path following.

The simulation results are compared and analyzed under different adhesion coefficients. With the decrease of pavement adhesion coefficient, the variation range of lateral deviation relative to the reference path increases. However, under the action of the controller, the lateral displacement deviation is timely corrected and finally converges to zero. The lateral deflection Angle and yaw velocity of the center of mass increase with the decrease of the road adhesion coefficient, and eventually tend to be stable. It indicates that under the action of the controller, the intelligent vehicle can resist the external interference in the path following process and has a good stability in the driving process, aiming at the working conditions of different vehicle speeds and different road adhesion coefficients.

\section{Acknowledgment}

Thanks for the foundation of NSFC (51505071) and Northeastern University.

\section{References}

[1] R.B.Wang, R.H.Zhang, F.You, et al. "Intelligent vehicle arc tracking control algorithm,'Journal of jilin university: engineering, vol. 36 (5), pp.731-735, 2006.

[2] H.Niu ,"Research on lateral motion control and monitoring of intelligent driving vehicles," Northeastern University, 2014

[3] X.M.Lin, "Design and simulation of intelligent vehicle path following control algorithm, "annual meeting proceedings of Chinese society of automotive engineering, pp. 390-393, 2014.

[4] Y.Song yan, P.Zhao, X.Tao, B.C.Li, H.W.Liang ,T. Mei," A cascade robust control method for path following of unmanned vehicles based on integrated muji, "ROBOT, vol. 35 (4) ,pp.417424,2013 . 
[5] G. Tagne, R. Talj, A. Charara," Higher-Order Sliding Mode Control for Lateral Dynamics of Autonomous Vehicles with Experimental Validation. Intelligent Vehicles Symposium," Gold Coast City, pp.678-683,2013. 